\title{
Hemorragia subaracnoídea no traumática con angiografía por tomografía computada inicial "Negativa"
}

\author{
Mario Zerega $R^{1}$, Karin Müller $C^{1}$, Rodrigo Rivera $M^{2}$, Sebastián Bravo $G^{3}$, Juan Pablo Cruz $Q^{3}$.
}

1. Residente de Subespecialidad en Neurorradiología Diagnóstica, Departamento de Radiología, Facultad de Medicina, Pontificia Universidad Católica de Chile. Santiago-Chile.

2. Servicio de Neurorradiología, Instituto de Neurocirugía Dr. Alfonso Asenjo. Santiago -Chile.

3. Departamento de Radiología, Facultad de Medicina, Pontificia Universidad Católica de Chile. Santiago-Chile.

\section{Non-Traumatic Subarachnoid Hemorrhage with "Negative” Initial Computed Tomography Angiography}

\section{Resumen}

La hemorragia subaracnoidea (HSA) no traumática es un subtipo de ictus hemorrágico que representa aproximadamente el $5 \%$ de todos los accidentes vasculares encefálicos (AVE). El $85 \%$ de los casos de HSA espontánea (no traumática) son secundarios a un aneurisma intracraneano roto, el 10\% a hemorragia perimesencefálica no aneurismática y el otro $5 \%$ a otras causas. Entre estas se incluyen malformaciones arterio-venosas, fístulas durales, vasculits, trombosis de vena cortical, síndrome de vasoconstricción reversible, angiopatía amiloidea y síndrome de encefalopatía posterior reversible.

La aproximación inicial a una HSA no traumática requiere un estudio angiográfico no invasivo con tomografía computada para la toma de decisiones terapéuticas. Si no se detecta un aneurisma sacular intradural que explique el sangrado, las conductas a seguir dependerán del patrón de distribución de la sangre. En esta revisión sugerimos una aproximación basada en 1) revisar el estudio inicial tomando en cuenta los puntos ciegos para la detección de aneurismas, 2) analizar el patrón de distribución de la sangre y 3) analizar los hallazgos en imágenes de acuerdo a las posibles causas según patrón.

Palabras clave: angiopatía amiloidea, angio TC, hemorragia subaracnoidea, vasculitis, vasoconstricción cerebral reversible, trombosis venosa cortical.

\begin{abstract}
Non-traumatic subarachnoid hemorrhage represents approximately $5 \%$ of strokes. From these, $85 \%$ of nontraumatic subarachnoid hemorrhage are secondary to a ruptured aneurysm, $10 \%$ to nonaneurysmal perimesencephalic hemorrhage and the other 5\% to other causes. These include but are not limited to arteriovenous malformations, dural fistulae, vasculitis, cortical vein thrombosis, reversible cerebral vasoconstriction syndrome, amyloid angiopathy and posterior reversible encephalopathy syndrome.

Initial workup of nontraumatic subarachnoid hemorrhage requires a non-enhanced CT and CT angiography for decision making and management. If there is no aneurysm as a source of hemorrhage, subsequent imaging studies will depend on blood distribution pattern. In this review we suggest an approach: 1) review blind spots for aneurysm detection in the initial CT angiography, 2) analyze blood distribution pattern and 3) evaluate imaging findings and possible causes according to each pattern.

Keywords: amyloid angiopathy, cortical vein thrombosis, CT angiography, reversible cerebral vasoconstriction syndrome, subarachnoid hemorrhage, vasculitis.
\end{abstract}

Zerega $M$, et al. Hemorragia Subaracnoídea no Traumática con Angiografía por tomografía computada inicial "Negativa". Rev Chil Radiol 2018; 24(3): 94-104.

Correspondencia: Juan Pablo Cruz Quiroga / jpcruz@med.puc.cl

Trabajo enviado el 07 de septiembre de 2018. Aceptado para publicación el 28 de septiembre de 2018.

\section{Introducción}

La hemorragia subaracnoidea (HSA) no traumática es un subtipo de ictus hemorrágico que representa aproximadamente el $5 \%$ de todos los accidentes vasculares encefálicos (AVE). El 80-85\% de los casos de HSA son secundarios a un aneurisma intracraneano 
roto y $10 \%$ a hemorragia perimesencefálica no aneurismática ${ }^{(1)}$. El $5 \%$ es secundario a otras causas ${ }^{(2)}$, incluyendo malformaciones arterio-venosas, fístulas durales, vasculits, trombosis de vena cortical y síndrome de vasoconstricción reversible.

La aproximación inicial a una HSA no traumática requiere un estudio angiográfico no invasivo con tomografía computada (TC) para la toma de decisiones terapéuticas y la estratificación de riesgo. En los casos en que la angio TC no muestra un aneurisma sacular intradural que explique el sangrado, las conductas a seguir dependerán del patrón de distribución de la sangre (aneurismático, perimesencefálico, de la convexidad).

El concepto de angio TC "negativa" no es sinónimo de un examen sin aneurismas visibles. Existen múltiples otras causas de HSA no traumática con hallazgos asociados en estudios de TC y resonancia magnética $(\mathrm{RM})^{(3)}$, los que pueden ser sutiles y requieren una sospecha clínica para mejorar su pesquisa diagnóstica por parte del radiólogo.

En esta revisión sugerimos una aproximación basada en 1) revisar el estudio inicial tomando en cuenta los puntos ciegos para la detección de aneurismas, 2) analizar el patrón de distribución de la sangre y 3) analizar los hallazgos en imágenes de acuerdo a las posibles causas según patrón.

\section{Puntos ciegos de aneurismas}

Como se dijo en la introducción, toda HSA no traumática debiera ser evaluada en forma inicial con una angio TC para buscar un posible aneurisma. La sensibilidad de la angio TC para la detección de aneurismas es de $98 \%$ con una especificidad cercana al $100 \%{ }^{(4)}$.

Las ubicaciones típicas de aneurismas sacula- res rotos son $(5,6): 1)$ complejo comunicante anterior $(\sim 30 \%), 2)$ arteria comunicante posterior ( 20\%), 3) "top" de la arteria basilar ( 15\%) y 4) arteria cerebral media $(12 \%)$. Otras ubicaciones incluyen bifurcación de la $\mathrm{ACl}$ y carótido oftálmico que son lugares más comunes en poblaciones con aneurismas no rotos.

Los falsos negativos en la interpretación de una angio TC son más frecuentes en las siguientes situaciones $^{(4,7):}$ 1) aneurismas pequeños $<3 \mathrm{~mm}$ en ubicaciones atípicas (Figura 1), 2) presencia de vasoespasmo, 3) aneurismas trombosados (Figura 2), aneurismas no saculares (aneurismas disecantes (Figura 3), aneurismas tipo "blíster" (Figura 4), aneurismas micóticos (Figura 5).

\section{Patrones de distribución de la HSA}

Una vez hecho el análisis del examen inicial, tomando en cuenta las causas de posibles falsos negativos en la interpretación, el segundo paso es estudiar el patrón de distribución de la HSA en la TC sin contraste. Existen tres patrones reconocidos (Figura 6): el perimesencefálico, el aneurismático, y el de la convexidad ${ }^{(8)}$. Cabe destacar que el patrón de distribución es válido sólo cuando la TC es realizada dentro de 72 hrs (preferentemente 24 hrs) después del ictus, ya que los mecanismos de circulación de LCR y absorción/degradación de la sangre pueden alterar esta distribución después de las $72 \mathrm{hrs}$.

\subsection{Patrón perimesencefálico}

La hemorragia perimesencefálica es una entidad clínico-radiológica que representa un subtipo de HSA no traumática criptogénica, de presentación clínica y curso benigno, con una baja tasa de vasoespasmo y resangrado. Definida como entidad por Van Gijn ${ }^{(9)}$, representa hasta un $68 \%$ de las HSA criptogénicas ${ }^{(10)}$.
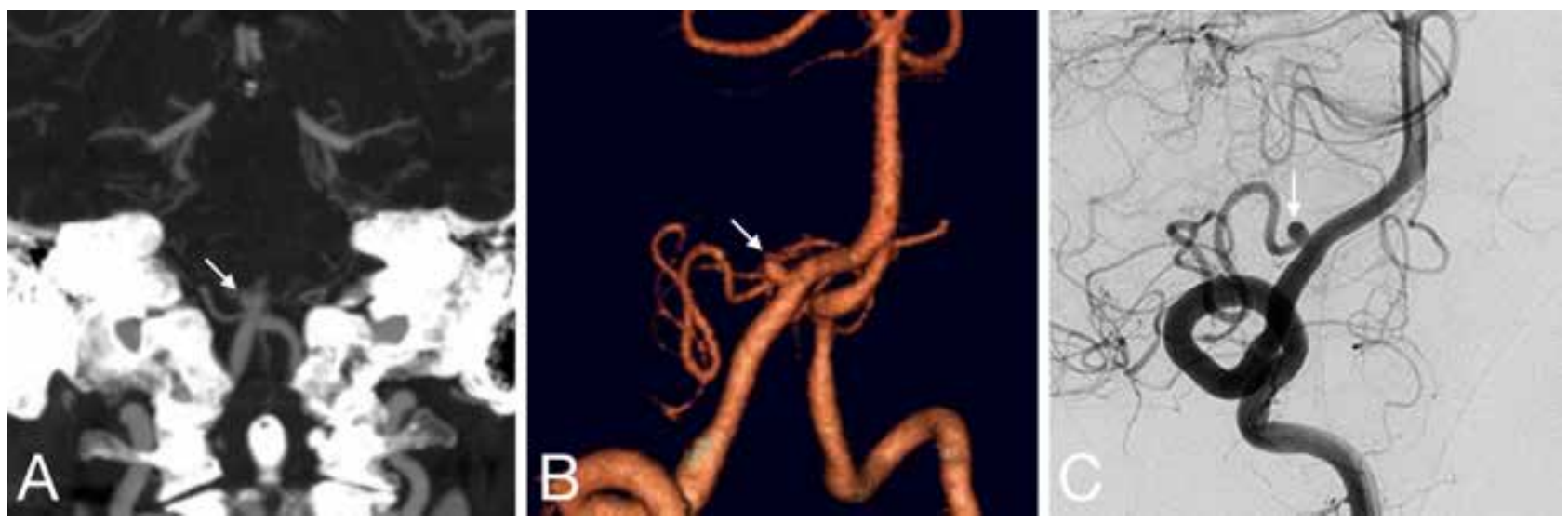

Figura 1: Pequeño aneurisma sacular de PICA derecha. Reconstrucciones MIP coronal (A) y MIP 3D (B) de angio TC. Proyección frontal de angiografía por sustracción digital $(C)$. Su pequeño tamaño y su ubicación atípica lo convierten en un potencial "falso negativo" de interpretación en angio TC. 

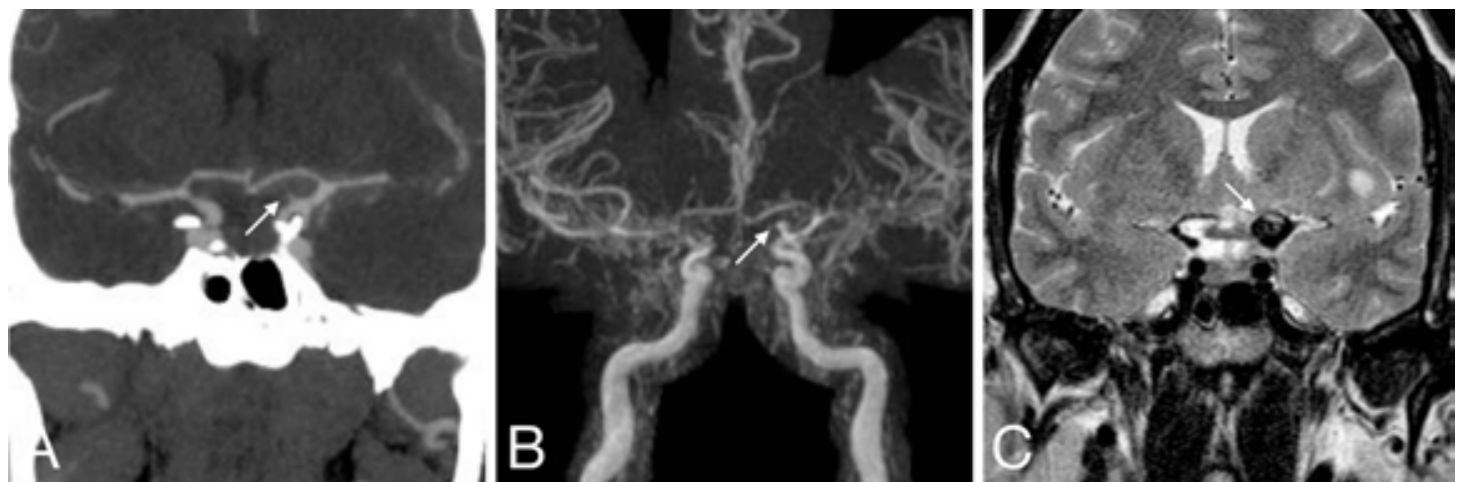

Figura 2: Aneurisma sacular parcialmente trombosado de ACI supraclinoidea izquierda. Reconstrucciones MIP coronal (A) de angio TC, MIP 3D de angio RM con contraste (B) y corte coronal T2 TSE de RM. La opacificación del aneurisma parcialmente trombosado es apenas visible en angio TC y evidente en angio RM. Sin embargo, ninguna de estas técnicas permite visualizar el saco aneurismático completo. La secuencia T2 TSE coronal de RM muestra un trombo predominantemente hipointenso en el interior del saco.
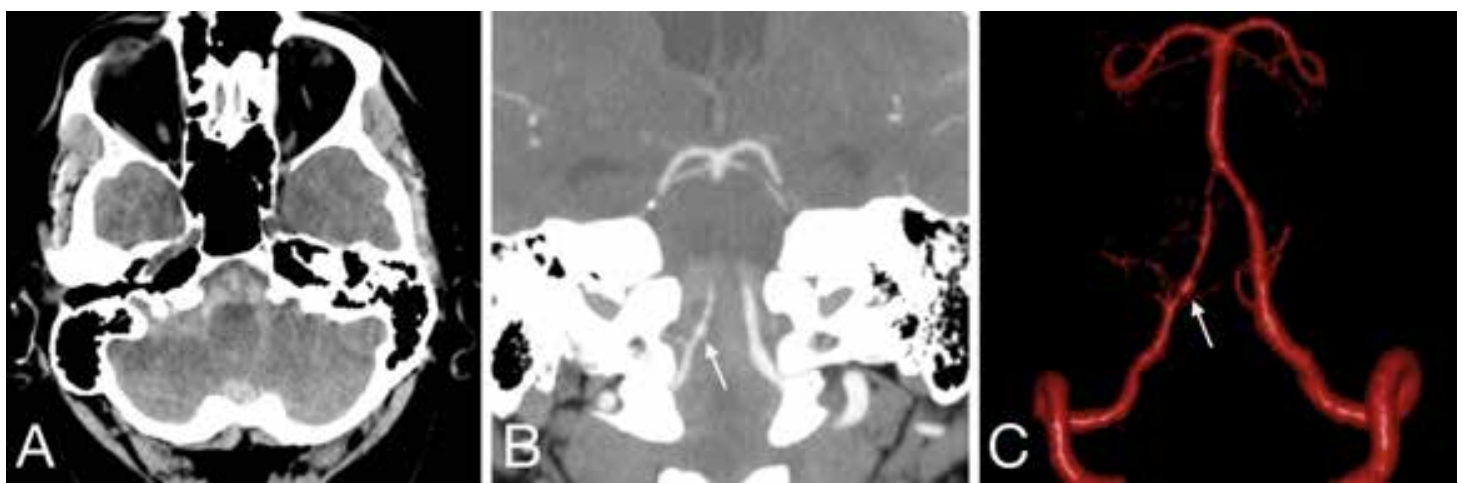

Figura 3: HSA secundaria a aneurisma disecante roto de arteria vertebral derecha. El corte axial (A) de TC muestra HSA en cisternas prebulbares. Las reconstrucciones MIP coronal (B) y MIP 3D de angio TC muestran HSA en fosa posterior y dilatación fusiforme alternante con áreas de estenosis de la arteria vertebral derecha intracraneana, hallazgos característicos de una disección transmural.
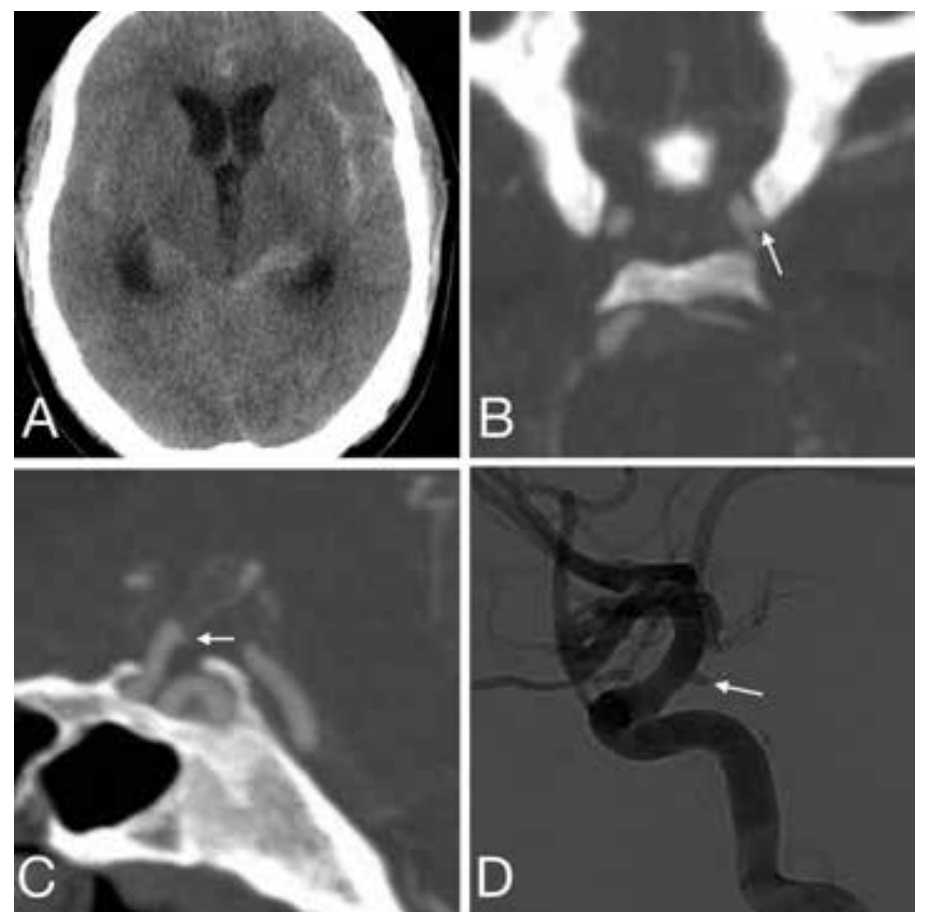

Figura 4: Aneurisma tipo "blíster" o ampolla. El corte axial (A) de TC muestra HSA en cisternas basales e hidrocefalia con dilatación ventricular y colapso de surcos de las convexidades. Las reconstrucciones axiales (B) y sagital (C) de angio TC muestran una saliente de perfil sésil de ACI clinoidea izquierda de superficie irregular con orientación lateral y caudal. 

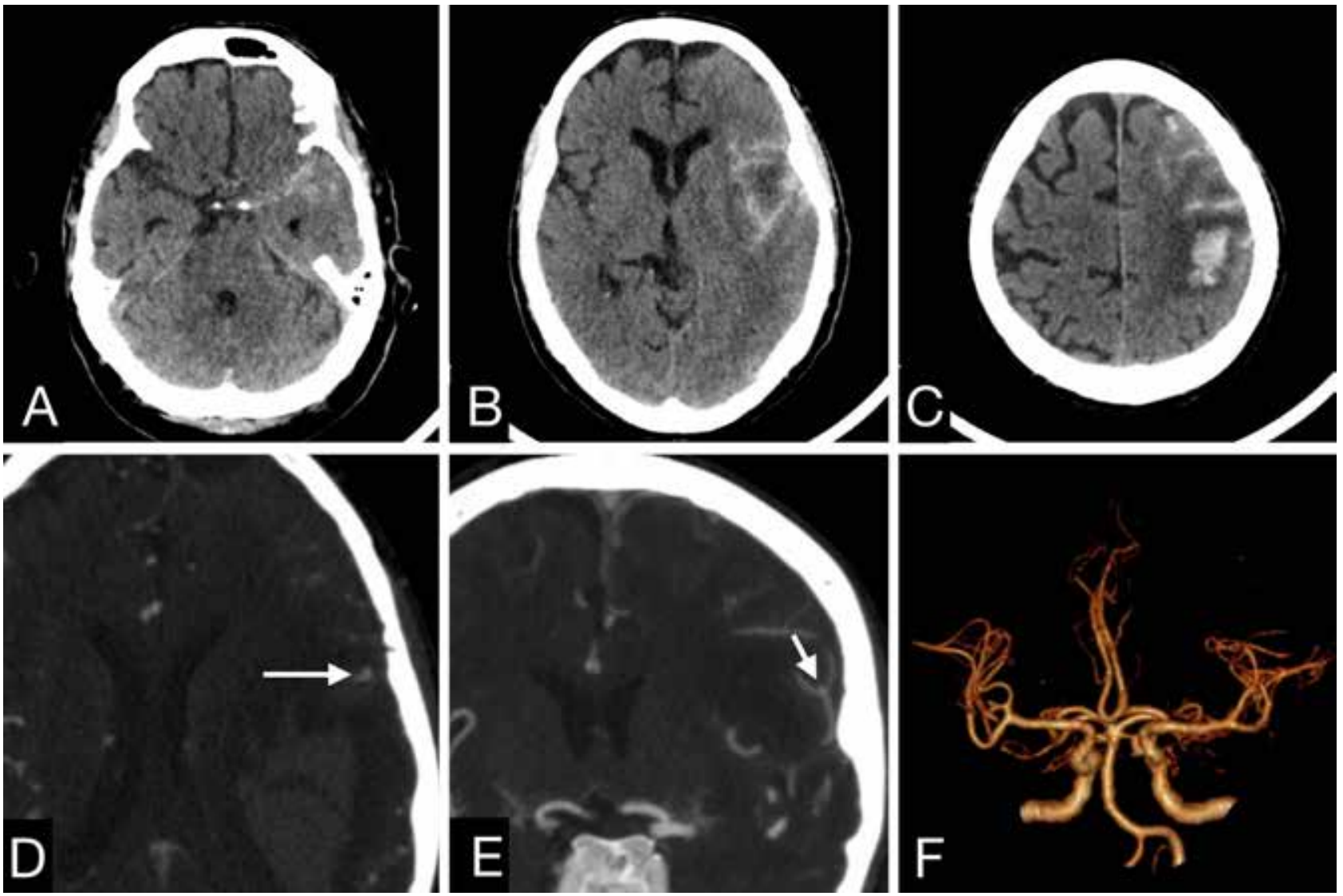

Figura 5: Los cortes axiales de TC $(A, B$ y $C)$ de un paciente con antecedente de endocarditis bacteriana muestran HSA de la convexidad y silviana izquierdas y un hematoma parenquimatoso frontoparietal ipsilateral. Las reconstrucciones axiales (D) y MIP coronal (E) de la angio TC muestran un pequeño aneurisma micótico roto de rama precentral de la división superior de ACM izquierda de $2 \mathrm{~mm}$. La reconstrucción MIP 3D muestra ausencia de aneurismas saculares proximales.
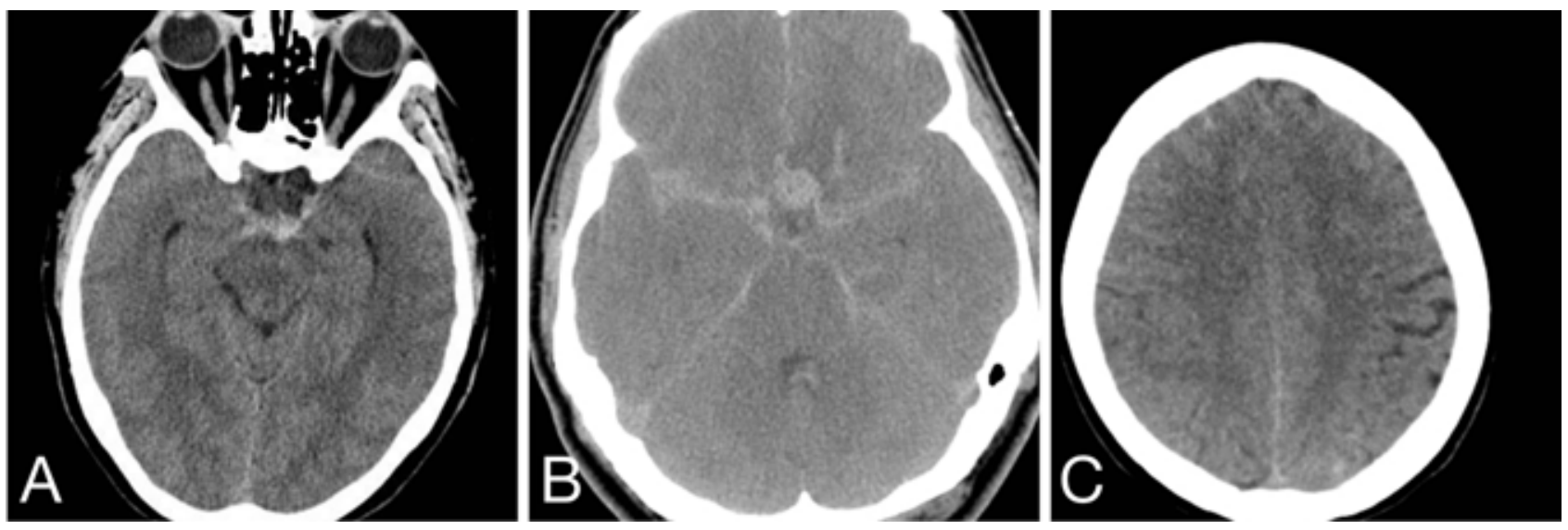

Figura 6: Patrones de distribución de la sangre en HSA no traumática. A patrón perimesencefálico. B patrón aneurismático. $C$ patrón de la convexidad. 
Para plantear el diagnóstico se requiere que el paciente esté en una buena condición clínica (GCS 15, WFNS 1, Hunt-Hess 1), y cumplir los siguientes criterios (Figura 7) ${ }^{(11,12)}$ :

1. Epicentro de la hemorragia inmediatamente anterior al mesencéfalo.

2. Extensión únicamente al aspecto posterior de la cisura interhemisférica.

3. Extensión únicamente al aspecto medial de las cisuras silvianas.

4. Ausencia de coágulo intraventricular. Se acepta escasa cantidad de sangre en porciones dependientes de los cuernos occipitales.

5. Ausencia de hematoma parenquimatoso.

6. Estudio angiográfico de buena calidad negativo.
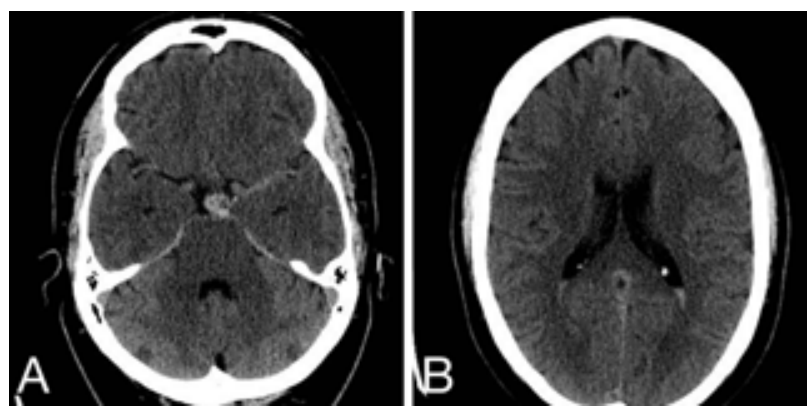

Figura 7: HSA de patrón perimesencefálico. A, la hemorragia está centrada anterior al mesencéfalo. $B$, se observa leve cantidad de sangre en las astas occipitales de los ventrículos laterales.

Si bien es aún controvertido, en estos pacientes se podría obviar la angiografía por sustracción digital
(ASD) diagnóstica, dado que los riesgos del procedimiento superan las probabilidades de encontrar un aneurisma intracraneano ${ }^{(13,14)}$, siempre y cuando se apliquen en forma estricta los criterios clínicos y de imágenes. Si se decide realizar ASD diagnóstica y esta es negativa, no es necesario realizar más exámenes, dado que los pacientes no se benefician de repetir los estudios tanto invasivos como no invasivos.

Pacientes que se presentan con hallazgos clínicos atribuibles a una mielopatía deben ser estudiados con una RM y angio RM cervical para descartar una fístula dural cervical. Esta puede simular una hemorragia perimesencefalica en imágenes (Figura 8)(15,16).

\subsection{Patrón aneurismático}

Un patrón aneurismático es cualquier HSA no traumática con compromiso de las cisternas basales que no cumpla con los criterios para un patrón perimesencefálico (Figura 9). Estos pacientes se deben considerar y manejar bajo el supuesto que tienen un aneurisma roto, por lo que realizar una ASD cerebral diagnóstica es mandatorio en este subgrupo. Hasta un $46 \%$ de estos pacientes tendrán un aneurisma en angiografía repetida a los 7 días y en 1/3 de estos casos el aneurisma era visible en estudio inicial(7). Tienden a ser aneurismas de $<3 \mathrm{~mm}$, aneurismas no saculares o en ubicaciones atípicas (Figura 1), como fue discutido anteriormente. En estos casos, si es que la primera angiografía es negativa, un estudio con RM puede mostrar un aneurisma trombosado, en el que el contraste no puede llenar el saco aneurismático, por ende, no se puede visualizar en angiografía (Figura 2) ${ }^{(17)}$.

La ASD permite descartar causas menos frecuentes como una pequeña micro-MAV, una micro-fístula pial superficial o una fístula dural.
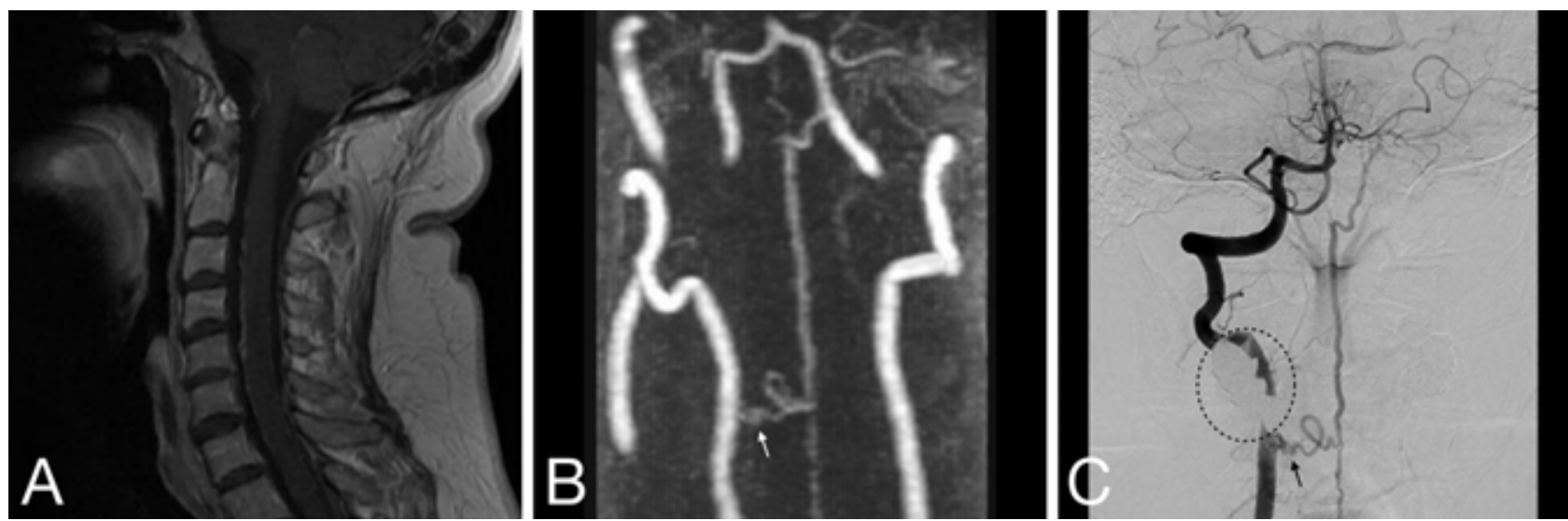

Figura 8: Fístula dural espinal cervical. A, en la RM de columna cervical la secuencia T1 sagital post contraste muestra dilatación y tortuosidad de la vena medular anterior. $B$, la reconstrucción $3 D$ de la angio $R M$ cervical con contraste en fase arterial muestra un cortocircuito arteriovenoso desde la arteria vertebral derecha a través de una arteria espinal segmentaria que determina opacificación precoz de la vena segmentaria correspondiente, que drena a la vena espinal anterior en la línea media. C, la angiografía por sustracción digital muestra el cortocircuito con mayor detalle anatómico (flecha negra). Se observa un artefacto secundario a la sustracción producida por tapaduras dentales tipo amalgama (elipse), que borra el medio de contraste endovenoso de la arteria vertebral. 
Todas estas entidades pueden dar un patrón aneurismático de HSA en TC ${ }^{(18)}$. Las causas extracraneanas son muy poco frecuentes (aneurisma de arteria espinal, MAV espinal, fístula dural de la unión cráneo cervical o cervical), pero deben ser sospechados cuando hay un exceso de sangre en el canal raquídeo y hay síntomas atribuibles a la médula espinal (Figura 10)(19).
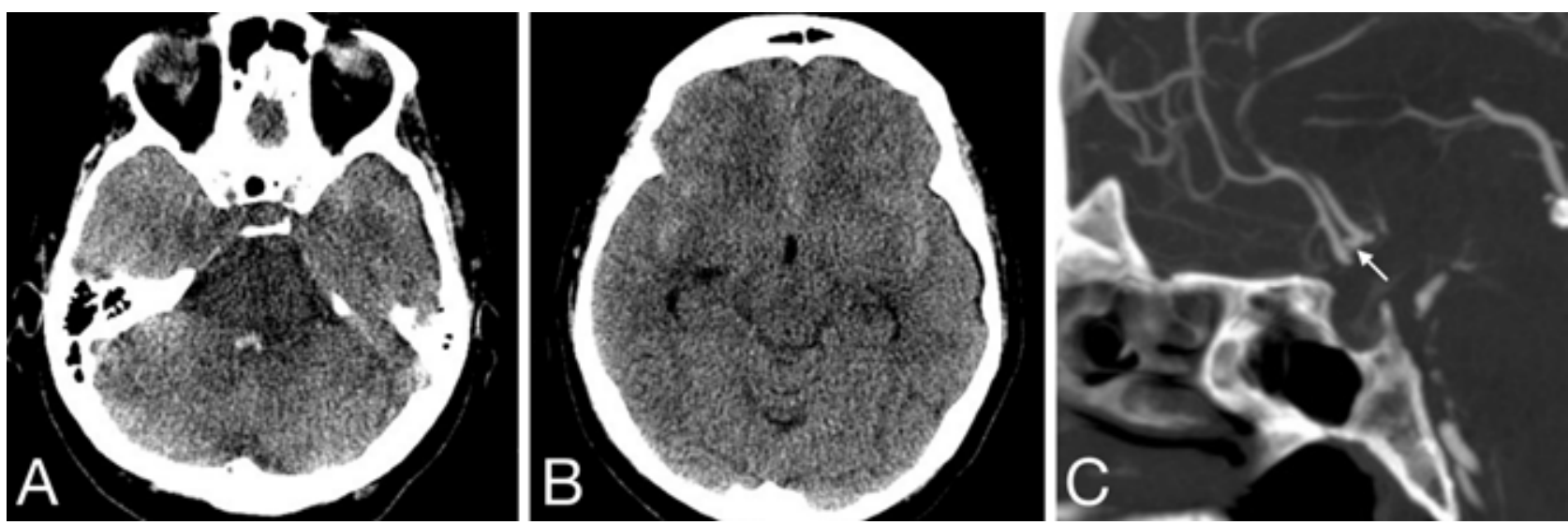

Figura 9: HSA de patrón aneurismático. Los cortes axiales de TC en fase sin contraste (A y B) muestran HSA en cisternas supraselar, silvianas, interhemisférica anterior, cisternas crurales e interpeduncular, contenido hemático en el IV ventrículo y cisternas prepontinas. La reconstrucción MIP sagital de angiografía por TC muestra un pequeño aneurisma de complejo comunicante anterior menor a $2 \mathrm{~mm}$ (flecha blanca).
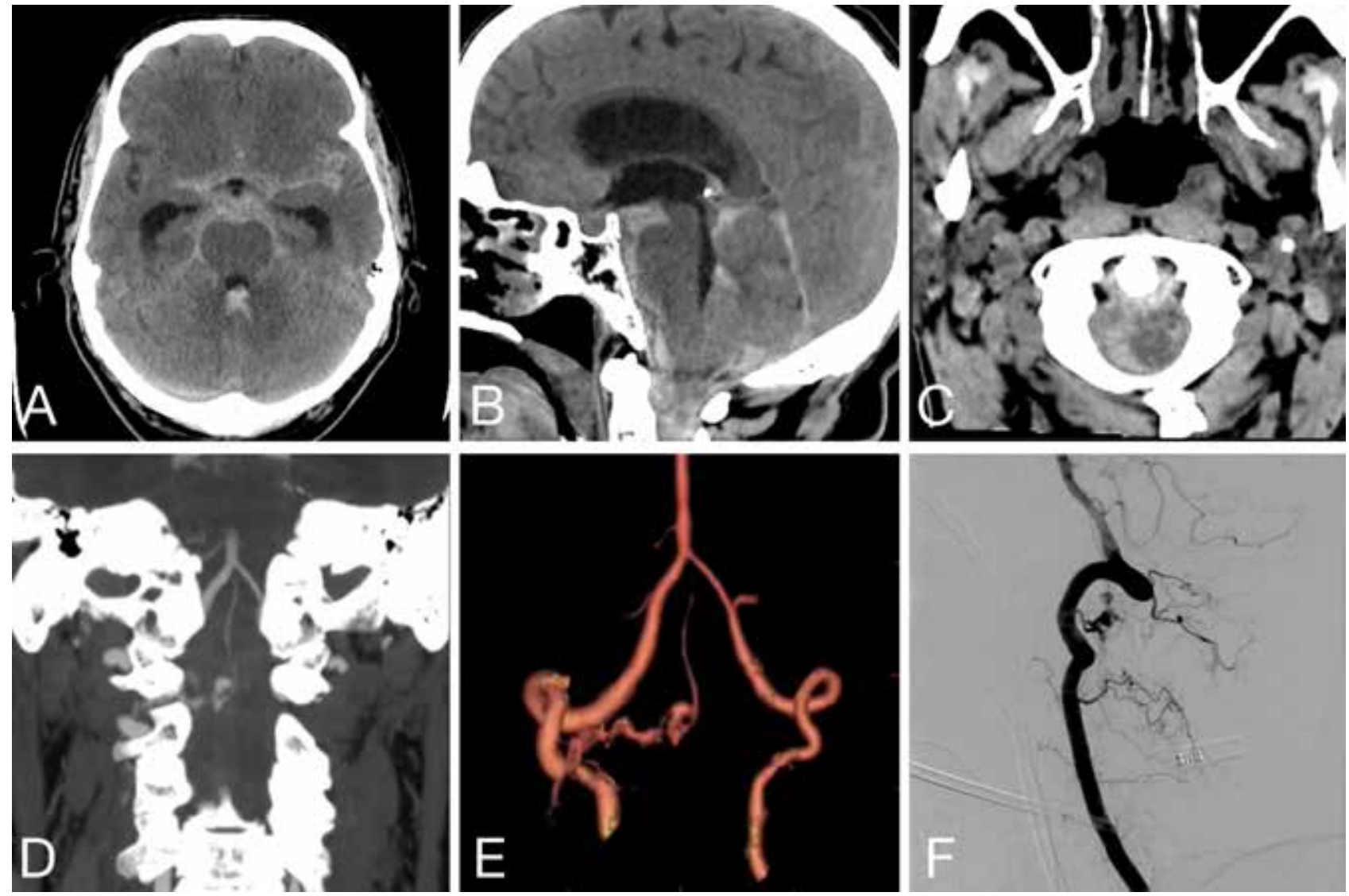

Figura 10: Los cortes axiales $(A, C)$ y sagital $(B)$ de tomografía computada sin contraste muestran una HSA de patrón aneurismático de predominio en la fosa posterior. A la altura de C1-C2 (C) se observa un coágulo en el canal raquídeo lateralizado a derecha, que desplaza la médula espinal hacia la izquierda. Las reconstrucciones MIP coronal (D) y MIP $3 D$ (E) de la angio TC muestran una fístula arteriovenosa de la unión cráneo cervical de tipo radiculopial C1-C2 derecha que es la causante de la HSA. 


\subsection{Patrón de la convexidad}

Se define como una HSA limitada a los surcos de la convexidad, sin compromiso de las cisternas basales. El diagnóstico diferencial es amplio y las enfermedades más relevantes que pueden dar este patrón incluyen:

- Síndrome de vasoconstricción reversible (SVCR). EI SVCR es una arteriopatía no inflamatoria que se presenta como manifestación clínica común a varias enfermedades, caracterizada por cefalea ictal, y vasoconstricción multifocal, que por definición se resuelve dentro de un periodo de 3 meses $^{(20)}$. La HSA de la convexidad es la manifestación hemorrágica más frecuente de este cuadro. La Angio TC y la ASD muestran estenosis multifocales de vasos pequeños ${ }^{(21)}$, que evoluciona con una migración centrípeta de las alteraciones durante la primera semana ${ }^{(22)}$. Los exámenes de control demuestran normalización del calibre a los 3 meses.

- Síndrome de Encefalopatía Posterior Reversible (SEPR). EI SEPR es un cuadro clínico caracterizado por encefalopatía asociado a zonas de edema parenquimatoso cerebral en zonas de territorio limítrofe, que predominan en el territorio posterior ${ }^{(23)}$. Pacientes con SEPR hipertensivo pueden presentarse con HSA de la convexidad en $5-17 \%$ por rotura de vasos piales debido a perdida de la auto regulación del flujo sanguíneo cerebral. Hallazgos asociados en TC incluyen hipodensidades multifocales bilaterales cortico subcorticales en territorio limítrofe superficial (parieto-occipitales, frontales superiores paramedianas), los cuales pueden ser mejor evaluados con RM, donde se identifica edema parenquimatoso, con hiperseñal en DWI y valores positivos en el mapa ADC (edema vasogénico), pudiendo existir áreas de edema citotóxico asociadas (valores bajos en el mapa ADC) $^{(24)}$ (Figura 11).

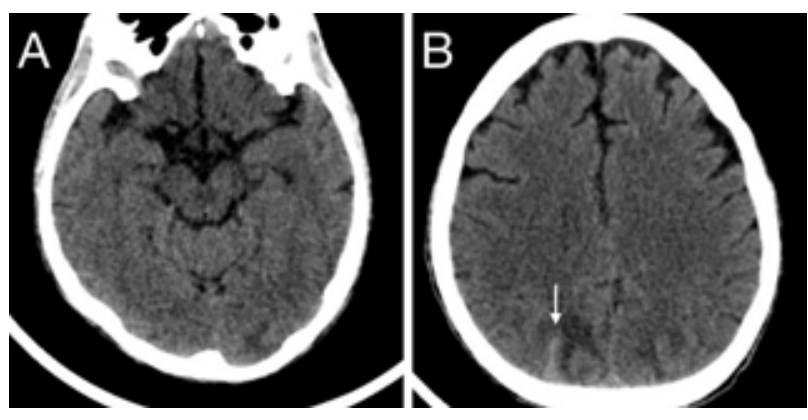

Figura 11: Síndrome de encefalopatía posterior reversible. Los cortes axiales de TC sin contraste (A y B) muestran hemorragia subaracnoidea en surcos occipitales y parietales, asociados a hipodensidad parenquimatosa cortico subcortical en las mismas áreas.
- Trombosis de vena cortical. La HSA sulcal es progresivamente reconocida como presentación de trombosis venosa cortical o de senos durales si bien el mecanismo no está claramente dilucidado(25). Un $6,4 \%$ se presentan con HSA aislada y un $4,6 \%$ tienen anormalidades parenquimatosas asociadas (edema cortico subcortical con o sin infarto hemorrágico)(26). Estas alteraciones son mejor evaluadas con RM por las ventajas intrínsecas de la resolución por contraste. En caso de dudas, el examen de elección es la venografía por RM con uso de medio de contraste ${ }^{(27)}$ (Figura 12).

- Vasculitis (primaria o secundaria) del SNC. Es un grupo heterogéneo y poco frecuente de HSA. Representan $<1 \%$ de las HSA de la convexidad. Tienen alteraciones del parénquima cerebral como microhemorragias, pequeños infartos y alteraciones de la sustancia blanca, mejor evaluadas por RM (Figura 13). Se pueden ver estenosis multifocales de vasos pequeños en angio TC o ASD, que no revierten a los 3 meses. La punción lumbar habitualmente está alterada (pleocitosis linfocitaria, aumento de proteínas), lo que ayuda al diagnóstico. Una PL y RM de cerebro normales tienen un alto valor predictivo negativo para vasculitis ${ }^{(28)}$. En este escenario, la ASD no contribuye mayormente al diagnóstico y el rol de la RM con protocolo para estudio de pared de vaso ha ganado importancia para la diferenciación de las arteriopatías no inflamatorias (p.ej. SVCR) de las arteriopatías inflamatorias ${ }^{(29)}$.

- Angiopatía amiloídea. Se caracteriza por depósito de proteína $B$ amiloide en la media y adventicia de vasos leptomeningeos y corticales, que debilita su pared y causa hemorragias. Su prevalencia aumenta con la edad, afectando a pacientes mayores de 55 años $^{(30)}$. En general es asintomática y cuando es sintomática más frecuentemente se presenta como hemorragias parenquimatosas lobares en pacientes no hipertensos (angiopatía amiloídea cerebral). Con menor frecuencia pueden existir cambios inflamatorios asociados al depósito de amiloide. Estos pueden ser de dos tipos: angiodestructivos (angeítis relacionada a $\mathrm{B}$ amiloide, con inflamación granulomatosa transmural) o perivasculares (reacción inflamatoria perivascular sin características angiodestructivas). En ambos casos la hemorragia parenquimatosa es menos frecuente y estos pacientes a menudo muestran cuadros de 

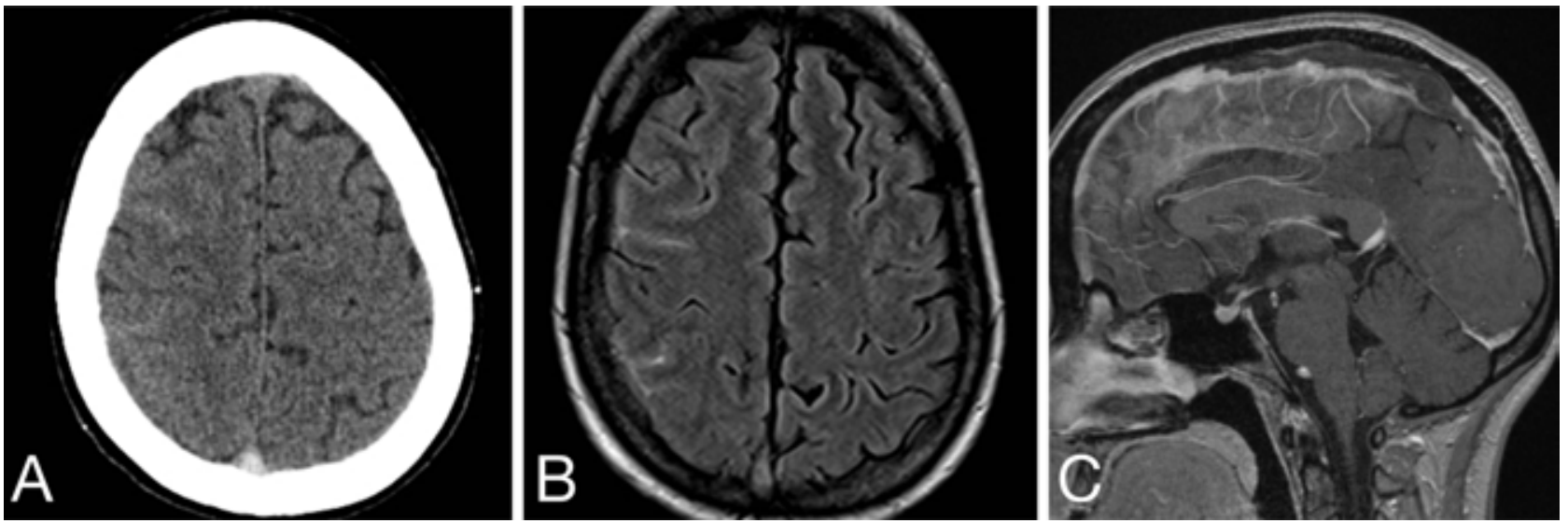

Figura 12: Trombosis venosa de seno sagital superior. Los cortes axiales de TC (A) y secuencia T2 FLAIR de RM (B) muestran HSA en surcos de la convexidad frontoparietal derecha, asociados a hiperdensidad (TC) y pérdida del vacío de flujo (FLAIR) del aspecto posterior del seno sagital superior. El corte sagital T1 gradiente post contraste de RM confirma un defecto de llenado en el seno sagital superior asociado a engrosamiento paquimeningeo e ingurgitación venosa.
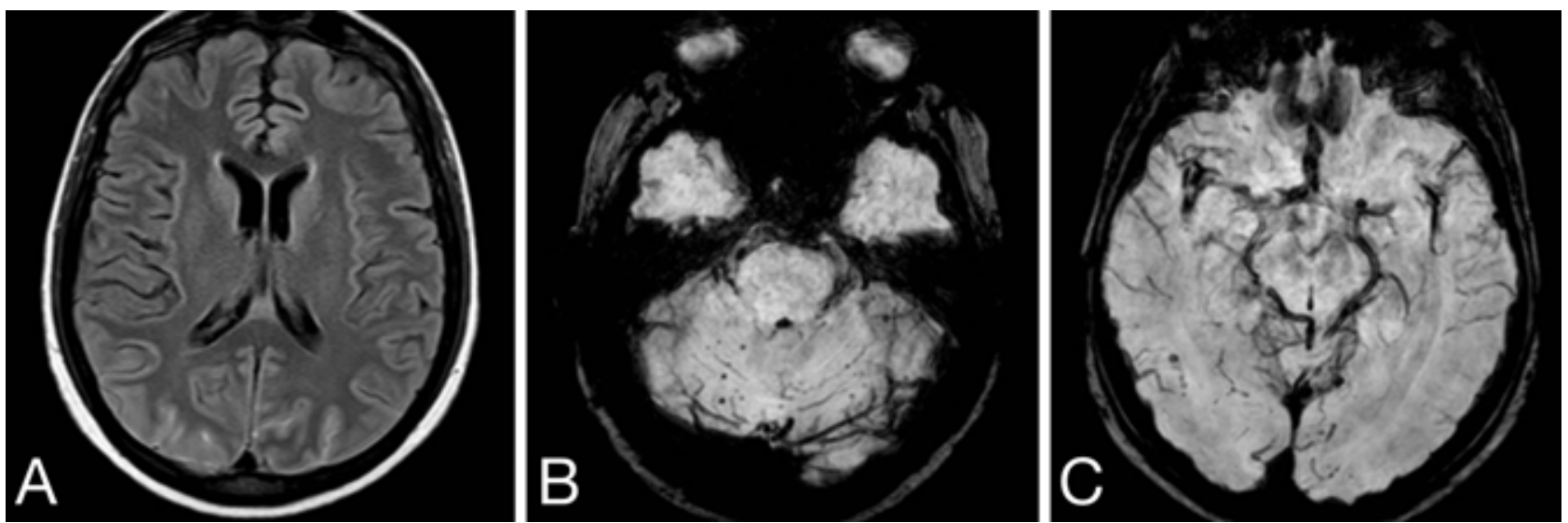

Figura 13: Vasculitis secundaria del SNC por lupus eritematoso sistémico. Los cortes axiales de secuencias T2 FLAIR (A) y susceptibilidad $(B$ y $C$ ) de RM muestran HSA en surcos occipitales y parietales, asociados a múltiples microhemorragias subcorticales cerebelosas y occipitales bilaterales.

deterioro cognitivo subagudo, convulsiones y cefalea, que responden a corticoides ${ }^{(31)}$. Hallazgos característicos en imagen son las microhemorragias lobares subcorticales detectables en secuencias de RM potenciadas en T2* (gradiente, susceptibilidad, BOLD), los hematomas parenquimatosos lobares (AA cerebral), siderosis superficial/hemorragia subaracnoidea en las convexidades ${ }^{(31,32)}$ con hipodensidad o aumento de señal T2 FLAIR cortico subcortical subyacente y refuerzo tras el uso de contraste (en pacientes con cambios inflamatorios asociados al depósito de B amiloide) (Figura 14).
- Uso de drogas recreacionales. El uso de drogas recreacionales habitualmente gatilla la rotura de una lesión pre-existente. Cualquier droga simpaticomimética puede inducir hipertensión y HSA de la convexidad. La cocaína es la más frecuente entre estas drogas y es un factor independiente de peor pronóstico en HSA. Los pacientes desarroIlan aneurismas en un 40-70\%, por ende, el patrón es habitualmente aneurismático ${ }^{(33)}$. Los pacientes también desarrollan vasculitis demostrada en estudios histológicos y de RM con estudio de pared de vaso ${ }^{(34)}$, lo que explica el patrón de la convexidad. 

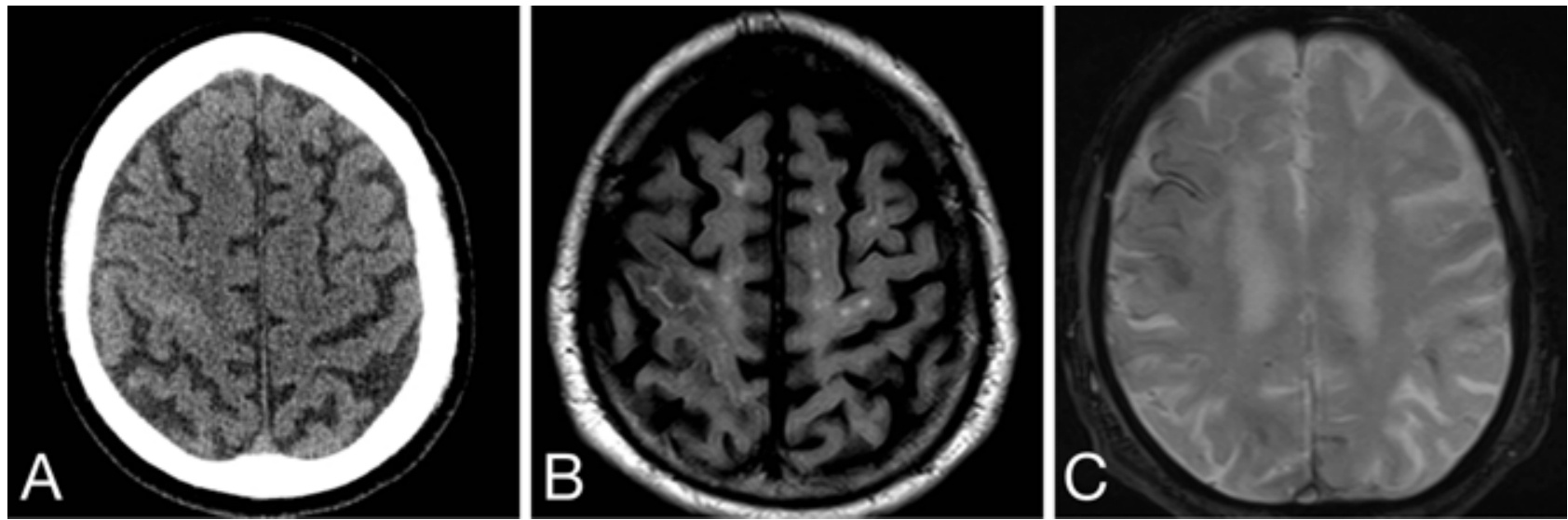

Figura 14: Angiopatía amiloidea con HSA de la convexidad. Los cortes axiales de TAC sin contrate (A) secuencias T2 FLAIR $(B)$ y $T 2^{*}(C)$ de RM muestran HSA en surco precentral derecho, con siderosis pial superficial frontoparietal bilateral, como manifestación de sangrados previos.

Algoritmo propuesto modificado de Agid et al ${ }^{(8)}$.

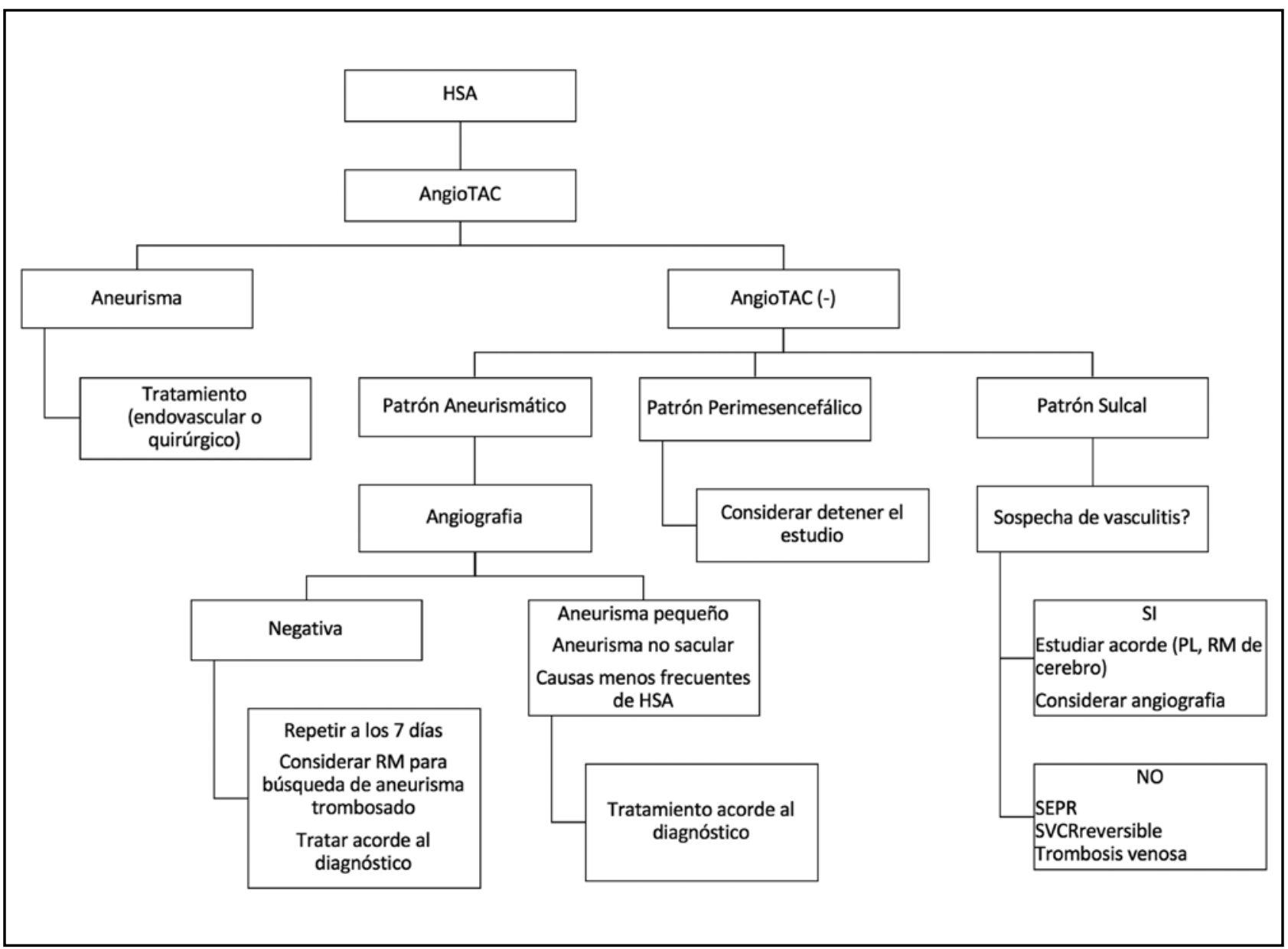

\section{Referencias}

1. Bederson JB, Connolly ES, Batjer HH, Dacey RG, Dion JE, Diringer MN, et al. Guidelines for the management of aneurysmal subarachnoid hemorrhage: A statement for healthcare professionals from a special writing group of the stroke council, American heart association. Stroke. 2009; 40(3): 994-1025.

2. Provenzale JM, Hacein-Bey L. CT evaluation of subarachnoid hemorrhage: A practical review for the radiologist interpreting emergency room studies. 
Emergency Radiology. 2009; 16: 441-451

3. Marder CP, Narla V, Fink JR, Tozer Fink KR. Subarachnoid hemorrhage: Beyond aneurysms. American Journal of Roentgenology. 2014; 202: 25-37.

4. Westerlaan HE, van Dijk JMC, van Dijk MJ, Jansenvan der Weide MC, de Groot JC, Groen RJM, et al. Intracranial aneurysms in patients with subarachnoid hemorrhage: CT angiography as a primary examination tool for diagnosis-systematic review and meta-analysis. Radiology. 2011; 258(1): 134-145.

5. Molyneux AJ, Kerr RS, Yu LM, Clarke M, Sneade M, Yarnold JA, et al. International Subarachnoid Aneurysm Trial (ISAT) of neurosurgical clipping versus endovascular coiling in 2143 patients with ruptured intracranial aneurysms: A randomised comparison of effects on survival, dependency, seizures, rebleeding, subgroups, and. Lancet. 2005; 366(9488): 809-817.

6. Forget TR, Benitez R, Veznedaroglu E, Sharan A, Mitchell $W$, Silva $M$, et al. A review of size and location of ruptured intracranial aneurysms. Neurosurgery. 2001; 49(6):1322-1325.

7. Jung JY, Kim YB, Lee JW, Huh SK, Lee KC. Spontaneous subarachnoid haemorrhage with negative initial angiography: A review of 143 cases. J Clin Neurosci. 2006; 13(10): 1011-1017.

8. Agid $\mathrm{R}$, Andersson $\mathrm{T}$, Almqvist $\mathrm{H}$, Willinsky RA, Lee SK, TerBrugge KG, et al. Negative CT Angiography Findings in Patients with Spontaneous Subarachnoid Hemorrhage: When Is Digital Subtraction Angiography Still Needed? Am J Neuroradiol. 2010; 31(4): 696-705.

9. van Gijn J, van Dongen KJ, Vermeulen M, Hijdra A. Perimesencephalic hemorrhage: a nonaneurysmal and benign form of subarachnoid hemorrhage. Neurology. 1985; 35(4): 493-497.

10. Flaherty ML, Haverbusch M, Kissela B, Kleindorfer D, Schneider A, Sekar P, et al. Perimesencephalic subarachnoid hemorrhage: Incidence, risk factors, and outcome. J Stroke Cerebrovasc Dis. 2005; 14(6): 267-271.

11. Schwartz TH, Solomon RA. Perimesencephalic nonaneurysmal subarachnoid hemorrhage: Review of the literature. Neurosurgery. 1996; 39(3): 433-440.

12. Kershenovich A, Rappaport ZH, Maimon S. Brain computed tomography angiographic scans as the sole diagnostic examination for excluding aneurysms in patients with perimesencephalic subarachnoid hemorrhage. Neurosurgery. 2006; 59(4): 798-801.

13. Cruz JP, Sarma D, Noel De Tilly L. Perimesencephalic subarachnoid hemorrhage: When to stop imaging? Emerg Radiol. 2011; 18(3): 197-202.

14. Mensing LA, Vergouwen MDI, Laban KG, Ruigrok YM, Velthuis BK, Algra A, et al. Perimesencephalic Hemorrhage: A Review of Epidemiology, Risk Factors, Presumed Cause, Clinical Course, and Outcome. Stroke. 2018; 1-9.

15. Hashimoto H, Lida JI, Shin Y, Hironaka Y, Sakaki T. Spinal durai arteriovenous fistula with perimesencephalic subarachnoid haemorrhage. J Clin Neurosci. 2000; 7(1): 64-66.

16. Wijdicks EFM, Schievink WI, Miller GM. MR imaging in pretruncal nonaneurysmal subarachnoid hemorrhage: Is it worthwhile? Stroke. 1998; 29(12): 2514-2516.

17. Rodriguez-Régent $C$, Edjlali-Goujon M, Trystram D, Boulouis G, Ben Hassen W, Godon-Hardy S, et al.
Non-invasive diagnosis of intracranial aneurysms. Diagn Interv Imaging. 2014; 95(12): 1163-1174.

18. Lasjaunias P, Chiu M, Ter Brugge K, Tolia A, Hurth M, Bernstein M. Neurological manifestations of intracranial dural arteriovenous malformations. J Neurosurg. 1986; 64(5): 724-730.

19. Hiramatsu M, Sugiu K, Ishiguro $T$, Kiyosue $H$, Sato $\mathrm{K}$, Takai K, et al. Angioarchitecture of arteriovenous fistulas at the craniocervical junction: a multicenter cohort study of 54 patients. J Neurosurg. 2017; 128(June): 1-11.

20. Miller TR, Shivashankar R, Mossa-Basha M, Gandhi D. Reversible cerebral vasoconstriction syndrome, part 1: Epidemiology, pathogenesis, and clinical course. Am J Neuroradiol. 2015; 36(8): 1392-1399.

21. Ducros A, Fiedler U, Porcher R, Boukobza M, Stapf C, Bousser MG. Hemorrhagic manifestations of reversible cerebral vasoconstriction syndrome: Frequency, features, and risk factors. Stroke. 2010; 41(11): 2505-2511.

22. Shimoda M, Oda S, Hirayama A, Imai M, Komatsu F, Hoshikawa K, et al. Centripetal propagation of vasoconstriction at the time of headache resolution in patients with reversible cerebral vasoconstriction syndrome. Am J Neuroradiol. 2016; 37(9): 1594-1598.

23. Bartynski WS. Posterior Reversible Encephalopathy Syndrome, Part 1: Fundamental Imaging and Clinical Features. Am J Neuroradiol. 2008; 29(6): 1036-1042.

24. Hefzy HM, Bartynski WS, Boardman JF, Lacomis D. Hemorrhage in Posterior Reversible Encephalopathy Syndrome: Imaging and Clinical Features. Am J Neuroradiol. 2009; 30(7): 1371-1379.

25. Oppenheim C, Domigo V, Gauvrit J-Y, Lamy C, Mackowiak-Cordoliani M-A, Pruvo J-P, et al. Subarachnoid hemorrhage as the initial presentation of dural sinus thrombosis. AJNR Am J Neuroradiol. 2005; 26(3): 614-617.

26. Boukobza M, Crassard I, Bousser MG, Chabriat $H$. Radiological findings in cerebral venous thrombosis presenting as subarachnoid hemorrhage: a series of 22 cases. Neuroradiology. 2016; 58(1): 11-16.

27. Farb RI, Scott JN, Willinsky R a, Montanera WJ, Wright $G$ a, TerBrugge KG. Intracranial venous system: Gadolinium-enhanced three-dimensional MR venography with auto-triggered elliptic centric-ordered sequenceinitial experience. Radiology. 2003; 226(1): 203-209.

28. Calabrese LH. Diagnostic strategies in vasculitis affecting the central nervous system. Cleve Clin J Med. 2002; 69(2): SII105-108.

29. Mandell DM, Mossa-Basha M, Qiao Y, Hess CP, Hui $F$, Matouk $C$, et al. Intracranial vessel wall MRI: Principles and expert consensus recommendations of the American society of neuroradiology. American Journal of Neuroradiology. 2017; 38: 218-229.

30. Linn J, Halpin A, Demaerel P, Ruhland J, Giese AD, Dichgans $M$, et al. Prevalence of superficial siderosis in patients with cerebral amyloid angiopathy. Neurology. 2010; 74(17): 1346-1350.

31. Salvarani C, Morris JM, Giannini C, Brown RD, Christianson T, Hunder GG. Imaging Findings of Cerebral Amyloid Angiopathy, A $\beta$-Related Angiitis (ABRA), and Cerebral Amyloid Angiopathy-Related Inflammation. Medicine (Baltimore). 2016; 95(20): e3613.

32. Moussaddy A, Levy A, Strbian D, Sundararajan S, Berthelet F, Lanthier S. Inflammatory Cerebral Amyloid 
Angiopathy, Amyloid- $\beta$-Related Angiitis, and Primary Angiitis of the Central Nervous System: Similarities and Differences. Stroke. 2015; 46(9): e210-213.

33. Chang TR, Kowalski RG, Caserta F, Carhuapoma JR, Tamargo RJ, Naval NS. Impact of acute cocaine use on aneurysmal subarachnoid hemorrhage. Stroke. 2013; 44(7): 1825-1829.

34. Abdel Razek AAK, Alvarez H, Bagg S, Refaat S, Castillo M. Imaging Spectrum of CNS Vasculitis. RadioGraphics. 2014; 34(4): 873-894. 